\title{
MODELO CONCEPTUAL PARA EL DESPLIEGUE DE PUBLICIDAD UBICUA SOPORTADO EN UN ESQUEMA DE COOPERACIÓN SMART TV - SMARTPHONE
}

\section{CONCEPTUAL MODEL FOR PERVASIVE ADVERTISING SUPPORTED ON A SMART TV - SMARTPHONE COOPERATION FRAMEWORK}

\author{
Francisco Martinez-Pabon', Gustavo Ramirez-Gonzalez², Ángela Chantre-Astaiza ${ }^{3}$
}

Fecha de recepción: 1 de agosto de 2013

Fecha de aprobación: 5 de marzo de 2014

Referencia: F. Martínez Pabón, G. Ramírez González, A. Chantre Astaiza. (2014). Modelo conceptual para el despliegue de publicidad ubicua soportado en un esquema de cooperación Smart TV - Smartphone. Ciencia e Ingeniería Neogranadina, 24 (1), pp. 116 - 142.

\section{RESUMEN}

La publicidad ha sido durante años una de las herramientas más valiosas del mercadeo a través de un enfoque principalmente masivo, generalizado y vertical entre clientes y anunciantes. No obstante, una nueva corriente conocida como publicidad ubicua marca una evolución en el concepto clásico hacia entornos más interactivos, personalizados y horizontales que busca mejorar la eficiencia y el impacto de la publicidad convencional. Gracias al apoyo de tecnologías emergentes que se sustentan en la evolución de los Smartphones y los Smart TV, el potencial de la publicidad ubicua es indudable, lo cual la ha convertido en un terreno fértil de investigación. El presente artículo presenta un modelo conceptual que condensa las áreas de investigación más relevantes relacionadas con el despliegue de publicidad en entornos de computación ubicua soportados en esquemas de cooperación Smart TV - Smartphone.

Palabras clave: publicidad ubicua, Smart TV, Smartphone

\section{ABSTRACT}

Advertising has been one of the most valuable marketing tools for years by means of a massive, wide-ranging and vertical approach between customers and advertisers. However, a new tendency known as pervasive advertising suggests an evolution of the

1. Ing. en Electrónica y Telecomunicaciones, mi grado profesional de MSc a PhD(c), Investigador Grupo de Ingeniería Telemática, fomarti@unicauca.edu.co, Universidad del Cauca, Popayán, Colombia.

2. Ing. en Electrónica y Telecomunicaciones, PhD., Profesor de planta, gramirez@unicauca.edu.co, Departamento de Telemática, Investigador Grupo de Ingeniería Telemática, Universidad del Cauca, Popayán, Colombia.

3. Administradora de Empresas, MSc, Investigadora Grupo de Desarrollo Turístico y Regional, achantre@unicauca.edu.co, Universidad del Cauca, Popayán, Colombia. 
classical concept towards a more interactive, customized, and horizontal environment that seeks to improve the impact and efficiency of conventional advertising. As a result of the support of emerging technologies related to the development of Smartphones and Smart TVs, there are no doubts about pervasive advertising potential and its value as a rich research field. This article introduces a conceptual model, which compiles the most relevant research areas related to pervasive computing applied to advertising supported on a Smart TV - Smartphone cooperation framework.

Keywords: pervasive advertising, Smart TV, Smartphone.

\section{INTRODUCCIÓN}

Sin lugar a dudas, la publicidad juega un papel importante como motor del comercio moderno en cualquiera de sus modalidades, al punto que grandes compañías como Google impulsan gran parte de sus ingresos en el sustento de la publicidad. Frecuentemente, el concepto de publicidad se confunde con el concepto de mercadeo, el cual es definido por la Asociación Americana de Marketing como "la actividad, conjunto de instituciones y procesos para crear, comunicar, entregar e intercambiar ofertas que tienen valor para los clientes, socios y la sociedad en general" [1]. Una revisión cuidadosa de esta definición sugiere el análisis de los cambios importantes que ha sufrido el mercadeo en su evolución a lo largo de la historia: el mercadeo de hoy se basa en una relación más horizontal y participativa entre las compañías y sus clientes, siendo estos últimos participantes activos en la concepción y despliegue de los productos en el mercado. Por otro lado, el mercadeo actual más que estar dirigido a los clientes per se, realmente se direcciona al conjunto de instituciones, comunidades y grupos que participan a lo largo de toda la cadena [2].

En este contexto, el mercadeo forma parte de un conjunto de instrumentos que usan las compañías para llegar a sus clientes directamente y que está relacionado con cuatro áreas específicas: producto, precio, logística y promoción [3]. Particularmente, la publicidad se enmarca en el contexto de la promoción y se define en términos simples como un proceso de comunicación masivo diseñado para cambiar la actitud y el comportamiento de los receptores [4]. Al respecto, Kotler y Keller [3] elaboraron una definición más precisa que conceptúa la publicidad como "cualquier forma remunerada de presentación y promoción de ideas, bienes o servicios por parte de un auspiciante identificado".

De acuerdo con las definiciones anteriores, uno de los aspectos clave en el concepto de publicidad es el grado de involucramiento de los consumidores con el producto o servicio. Su relevancia es crítica, dado que impacta directamente el comportamiento durante el proceso de decisión de compra. 
Como concepto de investigación, el grado de involucramiento fue introducido con el propósito de explicar el comportamiento de compra del usuario, teniendo en cuenta que el procesamiento de la información depende en gran parte de la relevancia de la información para el consumidor. En este sentido, este concepto hace referencia a la utilidad que se percibe sobre un producto basada en los intereses, valores y necesidades internas [5]. Desde este punto de vista, existen productos de alto involucramientoqueson degranimportancia para el consumidor y están estrechamente relacionados con riesgos financieros 0 sociales de alto impacto (por ejemplo, una casa o vehículo). Por otro lado, la mayoría de las compras generalmente son de bajo involucramiento y son percibidas como poco importantes (por ejemplo, alimentos), lo cual no conlleva a una búsqueda exhaustiva de información. Así, es generalmente válido en los esquemas de publicidad, que los productos de bajo involucramiento requieren poca información, pero ésta debe repetirse frecuentemente. Por su parte, los productos de alto involucramiento requieren el despliegue de más información, pero con una tasa de repetición menos frecuente [2].

En consecuencia, aunque la televisión, la radio o los periódicos han sido los medios tradicionales para el despliegue de publicidad por décadas, los sistemas de publicidad actuales han sido influenciados profundamente por el modelo de internet que ha dado paso a los esquemas online de publicidad [6]. De acuerdo con lo anterior, es posible distinguir cuatro componentes fundamentales: los anunciantes, los informantes, los clientes y los brokers (ver Figura 1).

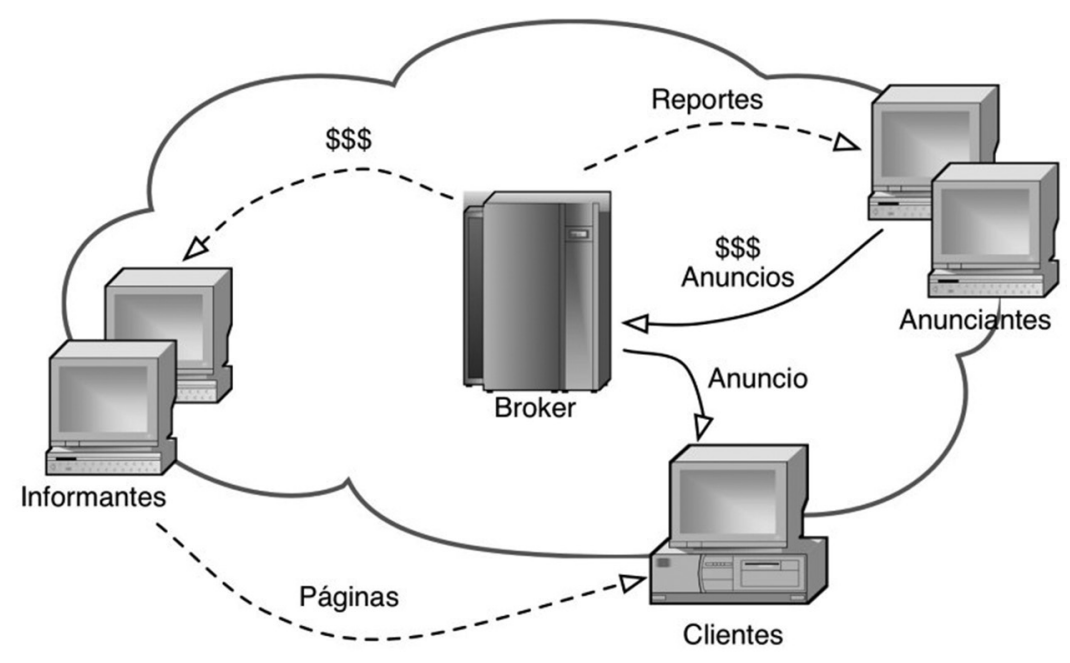

Figura 1. Modelo general de la publicidad en Internet.

Fuente: Haddadi et al. [6] 
Los anunciantes son aquellos que manifiestan su intención de vender sus productos y servicios a través de anuncios publicitarios; los informantes (por ejemplo, sitios de noticias, blogs, etc.) proveen espacios para ver los anuncios, por ejemplo a través de banners publicitarios; los clientes son los dispositivos que habilitan el despliegue de las páginas de los informantes y los anuncios respectivos; finalmente, los brokers (por ejemplo Google o Yahoo) proporcionan las plataformas para reunir a los anunciantes, los informantes y los clientes. En este sentido, los brokers proporcionan anuncios para los clientes, reúnen estadísticas sobre el comportamiento de los anuncios en las páginas, recaudan el dinero de los anunciantes y efectúan los pagos a los informantes.

Pasando a otro contexto, otra corriente interesante que marca la evolución de la publicidad actual tiene que ver con los medios masivos, los cuales están dirigidos a una audiencia de consumidores más amplia que no se encuentra en su sitio de residencia o trabajo, sino en sitios públicos o simplemente en tránsito mientras se dirige a un lugar en particular. Con frecuencia, las pantallas publicitarias o public displays pueden encontrarse en sitios cotidianos como aeropuertos, estaciones de tren o restaurantes [7]. Esta corriente, conocida como señalización digital (digital signage), ha merecido un foco de atención particular en los últimos años dado su gran potencial desde el punto de vista de mercadeo, si se tiene en cuenta que generalmente este tipo de pantallas se ubican cerca de los sitios de decisión de compra [8]. Recientemente, el modelo de Smart TV, entendido en términos simples como televisores o decodificadores con capacidades de cómputo conectados a la red [9], emerge como un nuevo ecosistema de pantallas conectadas y enriquecidas a través de aplicaciones que supone una nueva dimensión para la televisión interactiva y, por supuesto, con gran potencial para la señalización digital.

No obstante, pese a las nuevas oportunidades que encuentra la publicidad moderna en los esquemas de interactividad que marcan la evolución de internet y medios tradicionales como la televisión, aún el talón de Aquiles sigue siendo la ausencia de contenidos suficientemente personalizados yajustadosa laspreferencias delos usuarios. Por naturaleza, los medios tradicionales de publicidad son en esencia de emisión o transmisión (broadcast). En este contexto, la publicidad ubicua nace como un nuevo paradigma que redimensiona el modelo de la publicidad tradicional.

El presente artículo plantea un modelo conceptual que puede servir como referente para orientar las investigaciones relacionadas con el despliegue de publicidad ubicua soportado en un esquema de cooperación Smart TV - Smartphone. Con este propósito, la sección uno realiza una conceptualización general sobre la publicidad ubicua; la sección dos presenta algunas generalidades sobre el proceso que se abordó para la construcción del estado actual de conocimiento sobre la temática, incluyendo aspectos metodológicos; la sección tres presenta el modelo conceptual 
planteado, el cual incluye la identificación de las áreas de investigación más relevantes; y finalmente, en la sección cuatro se presentan algunas conclusiones.

\section{LA PUBLICIDAD UBICUA}

\subsection{UN NUEVO PARADIGMA EN LA PUBLICIDAD}

En términos simples, la publicidad ubicua se define como el uso de tecnologías de computación ubicua con fines publicitarios [10]. El concepto de computación ubicua se remonta a principios de los noventa cuando Marc Weiser concluye que "las tecnologías más profundas son aquellas que desaparecen. Ellas se entrelazan con nuestro diario vivir hasta hacerse indistinguibles" [11]: esa es la esencia de este nuevo paradigma que marca la evolución de la computación móvil. Por su parte, Boll et al. [12] agregan la siguiente conceptualización: "la computación ubicua describe la tendencia según la cual los dispositivos computacionales interconectados se entrelazan con los artefactos de nuestra vida diaria. Por consiguiente, el procesamiento, los sensores, la activación y la comunicación se encuentran embebidos en los dispositivos y ambientes, haciendo de la computación una parte integral de nuestra vida".

De acuerdo con lo anterior, la publicidad ubicua promueve una interacción con los usuarios en un nivel más íntimo y personalizado, que permite entregar información relevante en el momento y contexto adecuado [13]. En este sentido, los dispositivos móviles modernos (Smartphones) son claros candidatos para llenar los vacíos que aún enfrenta la publicidad tradicional y varios trabajos de investigación han abordado sus propuestas de publicidad ubicua con un claro soporte de las características que son inherentes a este tipo de dispositivos (disponibilidad de información de perfiles, interacción en redes sociales, sensores, localización, entre otras). No obstante, un esquema de cooperación Smart TV - Smartphone puede potenciar las fortalezas de ambos mundos y hacer frente a los desafíos de la publicidad moderna. El naciente modelo de Smart $T V$ no sólo es fuerte porque a diferencia de sus antecesores integra esquemas de computación y contenido distribuido (en la nube) de una forma optimizada para la televisión o la señalización digital, sino porque promueve un esquema de interacción simple con dispositivos como los Smartphones o tabletas. Este, sin lugar a dudas, es un valor agregado muy importante para el nuevo entorno generacional: "una generación conectada". Desde esta perspectiva, son muchas las oportunidades, pero también los retos que debe enfrentar la implementación de modelos de publicidad ubicua.

\subsubsection{Publicidad ubicua: oportunidades y retos}

En términos concretos, las oportunidades que ofrecen los modelos de publicidad ubicua se pueden resumir en los siguientes aspectos: i) transformación de los esquemas de broadcast tradicionales por esquemas interactivos; ii) personalización y 
adaptación al contexto; iii) mayor precisión en las medidas de audiencia y respuesta de los grupos objetivo; y iv) mecanismos de persuasión automatizada a través de la interacción social entre los usuarios [14]. No obstante, la publicidad ubicua también enfrenta una serie de retos que deben ser considerados: i) manejo adecuado del contexto de personalización: correcto emparejamiento entre necesidades (usuarios) y productos (ofertantes); ii) conveniente elección de los medios: para la entrega del contenido (SMS, video, mensaje instantáneo, banner, etc.) y el despliegue de la información (TV, Smartphone o ambos); iii) manejo apropiado del canal interactivo: capacidad del usuario para responder a los avisos publicitarios de diferentes maneras (solicitar más información, contactar un agente de ventas, comprar); y iv) privacidad: manejo confiable de la información de los usuarios y los mecanismos para la entrega de ofertas [13].

De acuerdo al planteamiento anterior, es claro que los modelos de publicidad ubicua pueden ser aún más efectivos que los modelos online, gracias a su potencial de personalización e interacción desde dispositivos portables como los Smartphones. Si a esto se suma el potencial de un esquema de colaboración con el modelo naciente de Smart TV, el impacto generado sobre el público objetivo puede ser aún mayor si se tienen en cuenta siete aspectos clave: i) combina las bondades de un medio masivo por excelencia como lo es la televisión; ii) agrega interactividad; iii) mejora la experiencia de usuario al facilitar la interacción a través de Smartphones, extendiendo las capacidades más allá del control remoto tradicional; iv) ofrece un modelo de conectividad y acceso a contenidos distribuidos (en la nube) como lo hacen los modelos online; v) redefine el modelo de publicidad ubicua tradicional al orientar contenidos no sólo a individuos sino también a grupos de individuos que comparten un espacio televisivo; vi) extiende sus fronteras al ofrecer la posibilidad de adaptar el contenido desplegado en más de una pantalla (aplicaciones n-screen) [15], de acuerdo con las capacidades de los dispositivos y el contexto específico; e igualmente, vii) los servicios que por naturaleza hacen parte de la telefonía móvil potencializan la experiencia del usuario (por ejemplo, al iniciar una llamada al servicio de ventas de forma inmediata).

\section{ESTADO ACTUAL DEL CONOCIMIENTO}

A continuación, se presenta el proceso de construcción de un modelo conceptual que sirve de base para trabajos de investigación relacionados con el despliegue de publicidad en entornos de computación ubicua soportados en esquemas de interacción Smart TV - Smartphone. En este sentido, la revisión del estado actual del conocimiento se abordó a través de una perspectiva de vigilancia tecnológica, con el objeto de establecer un panoramaglobal de la temática que plantea las bases para la definición de focos de investigación relevantes en el contexto de la publicidad ubicua. 


\subsection{METODOLOGÍA DE REVISIÓN}

En términos simples, la vigilancia tecnológica puede definirse como "la búsqueda, detección, análisis y comunicación de informaciones orientadas a la toma de decisiones sobre amenazas y oportunidades externas en el ámbito de la ciencia y tecnología" [16]. Para el propósito de la construcción del marco conceptual, la metodología para el desarrollo de la vigilancia tecnológica contempló las siguientes fases (Figura 2):

- Planeación: identifica las necesidades de información y el enfoque de la vigilancia tecnológica sobre el tema de interés. Durante esta fase, fue definido el objetivo de la vigilancia y se identificaron las fuentes de información más relevantes y las palabras clave que orientaron el proceso de búsqueda de información. En la Tabla 1, se presenta un resumen al respecto.

Haciendo referencia a las palabras clave, inicialmente se realizó una búsqueda preliminar utilizando un conjunto de cinco palabras clave: ubiquitous advertising, pervasive advertising, contextual advertising, pervasive computing, ubiquitous computing y Smart TV. A partir de un estudio preliminar de los resúmenes de los artículos arrojados por la búsqueda fue posible determinar que algunas palabras clave arrojaban resultados irrelevantes para el contexto de investigación. Luego de este primer filtro, se tomó la decisión de enfocar el análisis en los resultados de las búsquedas realizadas a partir de las palabras clave señaladas en la Tabla 1

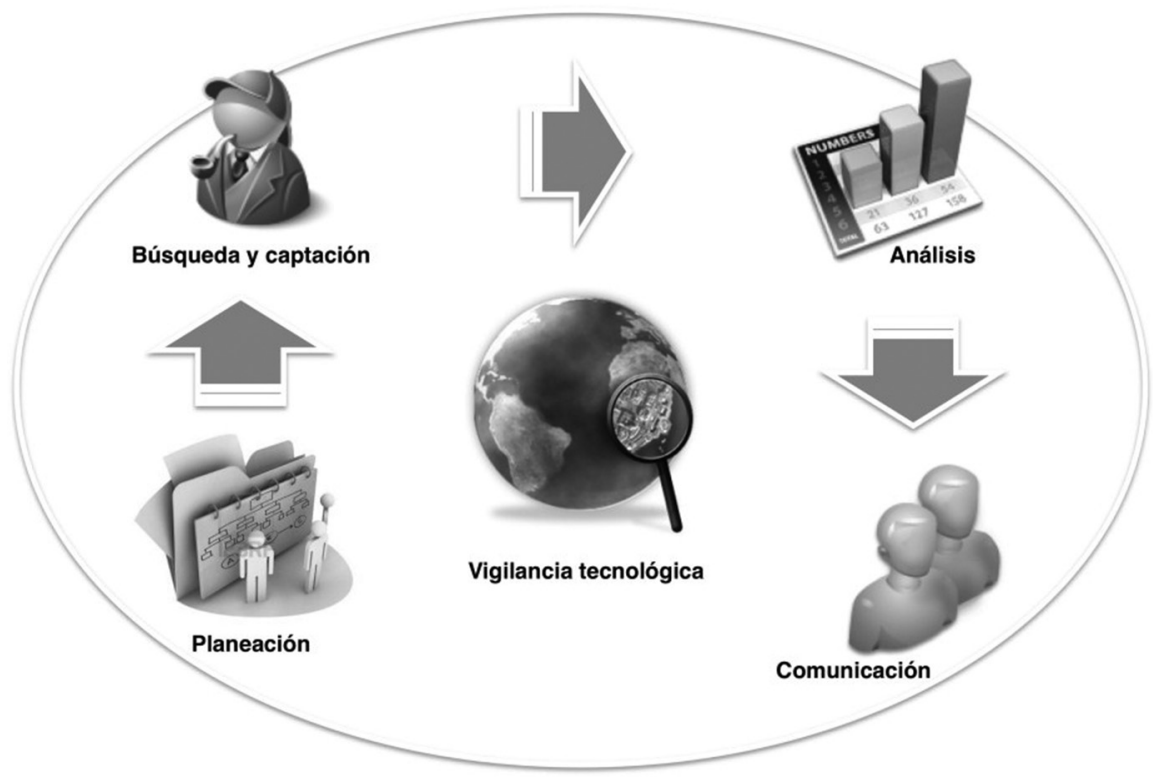

Figura 2. Metodología de la vigilancia tecnológica. 
Tabla 1. Planeación de la vigilancia tecnológica

\begin{tabular}{|l|l|}
\hline Objeto general del estudio & $\begin{array}{l}\text { Construir una visión general del estado actual del } \\
\text { conocimiento en el área de publicidad ubicua. }\end{array}$ \\
\hline \multirow{3}{*}{ Objeto específico del estudio } & $\begin{array}{l}\text { Identificar los autores más relevantes } \\
\text { Identificar las revistas y eventos más destacados en } \\
\text { la temática } \\
\text { Identificar los focos de investigación más relevantes }\end{array}$ \\
\hline \multirow{3}{*}{ Identificación de fuentes de información } & $\begin{array}{l}\text { Google Scholar } \\
\text { ACM Digital Library } \\
\text { IEEE Xplore Digital Library } \\
\text { Science Direct }\end{array}$ \\
\hline Palabras clave & $\begin{array}{l}\text { Pervasive advertising, ubiquitous advertising, } \\
\text { contextual advertising }\end{array}$ \\
\hline
\end{tabular}

- Búsqueda y captación: durante esta fase se realiza el proceso de búsqueda usando las fuentes de información identificadas, de acuerdo con los objetivos planteados en la fase anterior. El principal resultado de esta fase es la construcción de un corpus compuesto por los artículos más relevantes sobre la temática del proyecto, que constituyen el insumo básico para el análisis que se realizará en la siguiente fase. En total se identificaron más de 200 artículos.

- Análisis: en esta fase se realiza un análisis exhaustivo de la información del corpus construido en la fase anterior de acuerdo con los objetivos planteados para el esquema de vigilancia tecnológica. Para este propósito, se tuvo el soporte de dos paquetes de software: Matheo Analyzer y Publish or Perish. Matheo analyzer es un software de apoyo a la toma de decisiones que permite crear un cuadro de mando con mapas de información, síntesis e indicadores a partir de grandes conjuntos de información [17]. Por otro lado, Publish or Perish es un software libre que permite conocer el impacto de las publicaciones a través de la información que se obtiene de Google [18]. A manera de demostración sobre algunos resultados descriptivos, la Figura 3 resume autores relevantes identificados, la Figura 4 muestra algunos de los artículos más citados $y$, finalmente, la Figura 5 condensa algunas conferencias de interés.

- Comunicación: el objetivo de esta fase es socializar los resultados obtenidos durante el proceso de análisis realizado en la vigilancia tecnológica, resultados que han sido condensados en el presente artículo. 


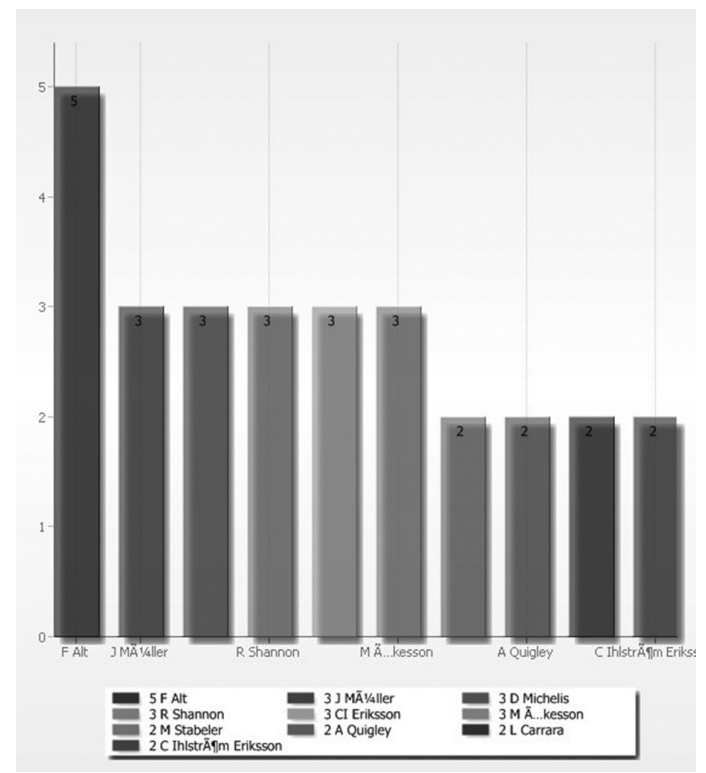

Figura 3. Autores más relevantes.

Fuente: Matheo Analyzer

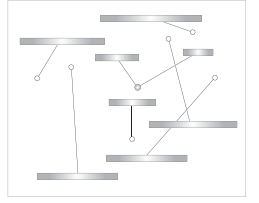

PERVASIVE SYMBIOTIC ADVERTISING SYSTEM AND METHODS THEREFOR

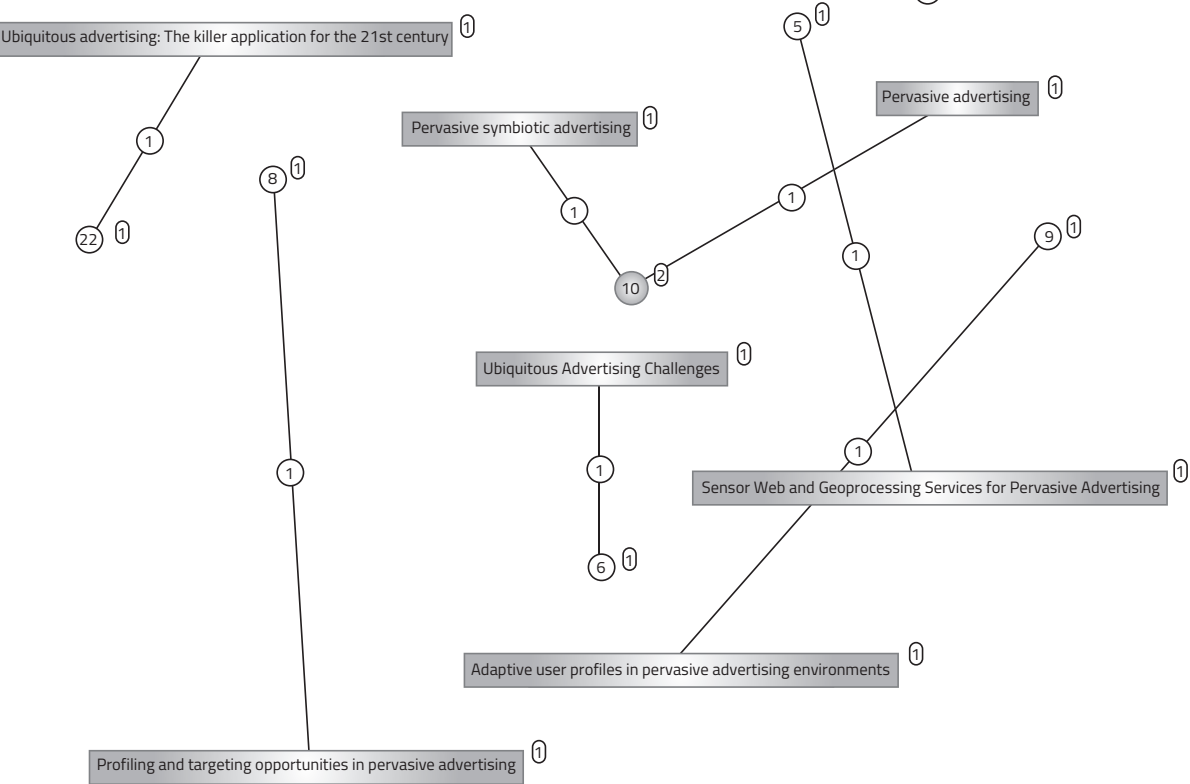

Figura 4. Artículos más citados.

Fuente: Matheo Analyzer. 


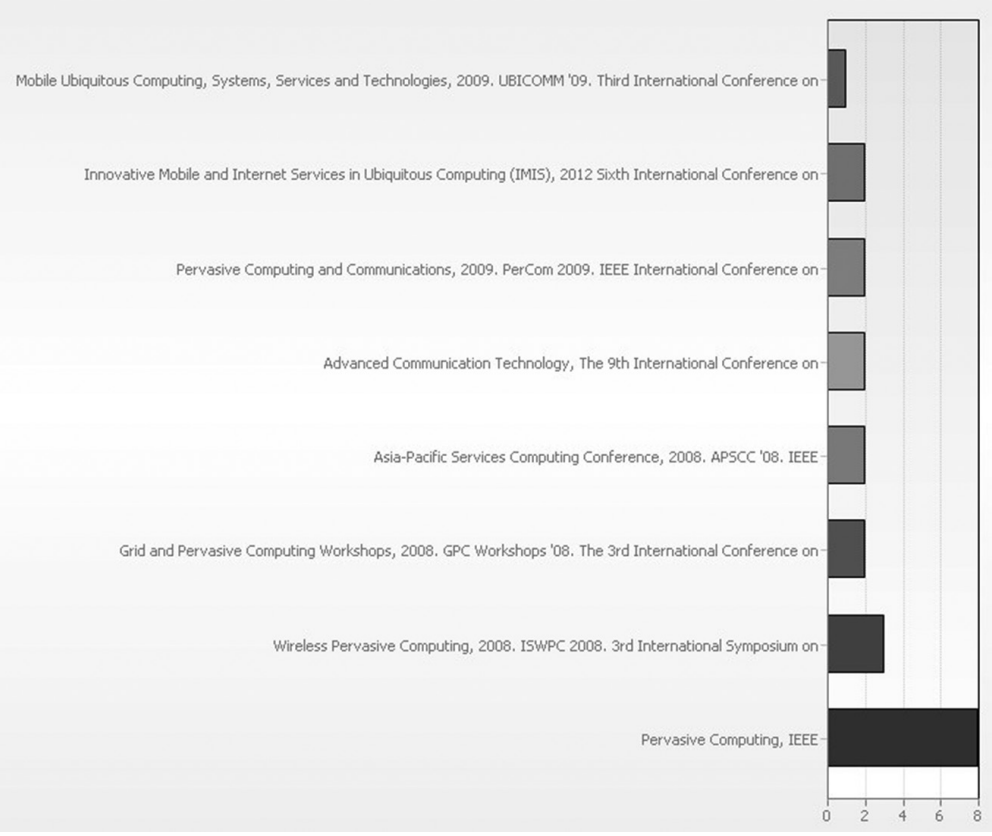

Figura 5. Revistas y conferencias más relevantes.

Fuente: Matheo Analyzer.

\subsection{CONCLUSIONES PRELIMINARES DEL PROCESO DE VIGILANCIA TECNOLÓGICA}

De acuerdo a los resultados encontrados durante el proceso, es posible plantear las siguientes conclusiones preliminares:

- Aunque la publicidad ubicua es un área de investigación que se ha venido cultivando desde hace varios años, el contexto de investigación planteado, que además considera un entorno de cooperación de los Smartphones con el naciente modelo de Smart TV, ha sido poco explorado. Como soporte a esta evidencia, la mayoría de trabajos relacionados han sido publicados en eventos más que en revistas especializadas.
- Un análisis más profundo de las publicaciones más relevantes y los trabajos de los autores más destacados demuestra que existe un énfasis importante en el área de señalización digital y el uso de Smartphones con fines de publicidad ubicua, pero no se considera un contexto propiamente dirigido a la incorporación del modelo de Smart TV.

- Igualmente, se puede inferir que existen dos contextos bien definidos para abordar un modelo de cooperación Smart TV - Smartphone con fines de publicidad ubicua:

- Señalización digital, contexto en el cual el modelo de Smart TV puede 
ampliar el potencial existente gracias a las capacidades de cómputo y mecanismos de interacción (sensores) que incorporan estos dispositivos sobre las viejas pantallas.

- Social TV, contexto que describe a un conjunto de personas viendo la televisión e interactuando entre sí y/o con amigos cercanos en un entorno social, usando sus Smartphones. En este sentido, las compañías publicitarias podrían tomar ventaja de esta interacción social para entregar ofertas más personalizadas usando en conjunto no sólo las capacidades de los Smartphones, sino también de los Smart TV.

\section{PLANTEAMIENTO DEL MODELO CONCEPTUAL}

Gracias a los resultados del proceso de vigilancia tecnológica y una revisión más exhaustiva de los trabajos más relevantes identificados durante el proceso fue posible plantear un modelo conceptual que evidencia las principales áreas de investigación en lo que respecta a la publicidad ubicua soportada en esquemas de cooperación Smart TV - Smartphone. Específicamente, el modelo describe los planteamientos más importantes en cada área y posibles brechas que pueden convertirse en un insumo importante para la formulación de futuras propuestas de investigación. La Figura 6 muestra un diagrama básico del modelo conceptual planteado.

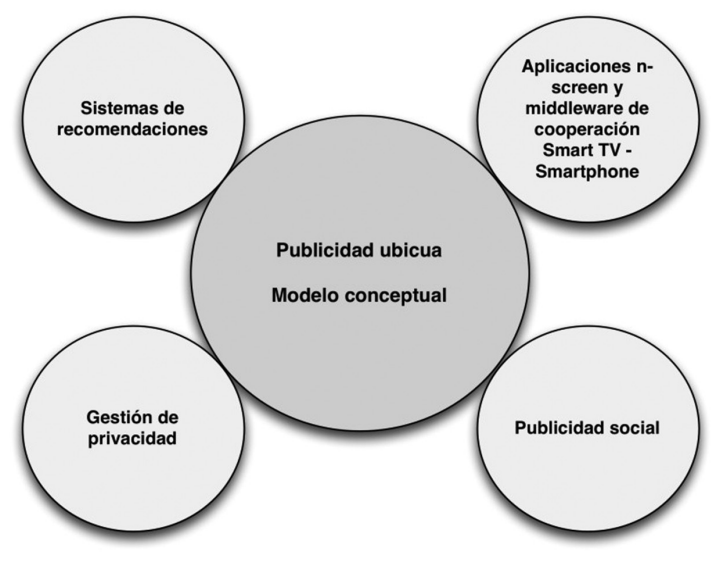

Figura 6. Modelo conceptual

Como se puede observar, el modelo cooperación Smart TV - Smartphone, conceptual plantea cuatro áreas de gestión de privacidad y publicidad social. investigación a saber: Sistemas de A continuación se realizará una revisión recomendaciones para publicidad ubicua, más detallada de cada una de las áreas de aplicaciones n-screen y middleware de conocimiento identificadas. 


\subsection{SISTEMAS DE RECOMENDACIONES PARA PUBLICIDAD UBICUA}

En términos simples, los sistemas de recomendaciones (SR) son herramientas software y técnicas que proporcionan sugerencias sobre ítems de interés para un usuario [19-21]. En términos más rigurosos, los SR basan la esencia de su funcionamiento en el siguiente planteamiento: si se considera que $C$ es el conjunto de usuarios del sistema e I representa el conjunto de ítems que pueden ser recomendados, el número de elementos de los dos conjuntos puede ser muy grande, luego es necesario reducir el número de recomendaciones para disminuir la carga cognitiva del usuario a través de una función de utilidad $u$ : $C \times I \rightarrow R$. Esta función mide el grado de satisfacción del usuario hacia un ítem, así que el sistema debe escoger ítems que maximicen la utilidad. El problema central de los SR radica en que $u$ no está definida completamente en $C \times I$, sino en algunos subconjuntos del espacio. En otras palabras, el usuario no ha expresado su preferencia o rating para cada ítem, lo cual sugiere que una de las principales funciones de un SR es la de predecir los ratings para ítems no evaluados, de tal manera que se pueda maximizar la utilidad $u$ [22].

En este sentido, diversas aproximaciones se han diseñado para mejorar la funcionalidad de los SR, entre las cuales se encuentran: i) filtrado colaborativo, que procesa correlaciones entre usuarios para reconocer su afinidad y asociar sus evaluaciones sobre los ítems; ii) filtrado basado en contenido, que usa las preferencias del usuario actual y predice los ratings con base en el grado de similitud con ítems que se han evaluado en el pasado; y iii) filtrado basado en comunidad, que realiza recomendaciones de acuerdo con las preferencias de los amigos del usuario.

En el contexto de la publicidad ubicua, los sistemas de recomendaciones juegan un papel muy importante por cuanto son la base para empezar a construir recomendaciones personalizadas y disminuir el esfuerzo cognitivo requerido por el usuario para asimilar la información y mejorar su grado de involucramiento con la oferta. Diversas investigaciones han abordado los problemas comunes a los sistemas de recomendaciones tradicionales desde diferentes perspectivas: los sistemas basados en contenido y filtrado colaborativo, sufren la existencia del problema del usuario o ítem nuevo (cold start) cuando no se tiene la cantidad de ratings suficientes para realizar la recomendación; al respecto, [23-24] proponen algunas técnicas que pueden ser complementadas con la utilización de filtros basados en comunidad, según lo sugieren las investigaciones de Golbeck [25] y Massa et al [26]. Otros problemas, relacionados con la dispersión de los datos (pocos ratings para gran parte de los ítems disponibles), han sido abordados en [27-30]; por otro lado, la combinación de los diferentes tipos de filtrado para robustecer las capacidades del SR, conocido como filtrado híbrido, ha sido ampliamente estudiada a través del empleo de diferentes técnicas Burke [21], Pazzani [27], [31-36].

No obstante, los SR para publicidad ubicua agregan nuevos retos a los focos de investigación que tradicionalmente 
se han abordado. En primer lugar, como fue señalado anteriormente, los SR convencionales operan en un espacio bidimensional Usuario x Ítem. Sin embargo, la publicidad ubicua requiere la incorporación de información del contexto que debe ser tenida en cuenta al momento de formular la recomendación, lo cual sugiere la ampliación del espacio tradicional Usuario $x$ Ítem a un espacio Usuario $x$ Ítem $x$ Contexto. Al respecto, Kuflik et al [37], realiza algunos aportes importantes para el modelamiento del contexto del usuario en entornos más generales, mientras que Heckmann et al [38] va un paso más allá y define una metodología para la caracterización del contexto en entornos de publicidad ubicua; en el mismo sentido, Hussein et al [39] examina algunas técnicas para la incorporación de información contextual en los SR y Carolis [40] plantea la incorporación de algunos datos relacionados con el contexto de localización en un entorno de publicidad. Adicionalmente, aunque la información contextual aporta elementos relevantes, el enriquecimiento de los perfiles es clave para propósitos de publicidad, más allá de la disponibilidad de ratings Partridge et al [41].

Por otro lado, generalmente los SR dirigen sus recomendaciones a individuos; no obstante, en el campo de la señalización digital por ejemplo. se requiere que las recomendaciones estén dirigidas a un grupo de personas que se encuentran observando la pantalla. Al respecto, Masthoff [42] realiza una revisión de algunas técnicas para la caracterización de un perfil grupal; igualmente, algunos sistemas han sido diseñados para entornos grupales: [43-52]. Específicamente, en el campo de publicidad, Carolis [40] plantea una aproximación para la caracterización y el modelamiento de los grupos, con el fin de entregar recomendaciones al conjunto de individuos. Sin embargo, el esquema de interacción tiene un componente fuertemente explícito, algo susceptible de mejorar en entornos computación ubicua. En ese orden de ideas, la inclusión de información contextual luce como un campo de investigación atractivo para los RS en este caso. Aunque el concepto de contexto es bastante amplio y puede incluir información que va desde la localización del usuario hasta datos de su círculo social cercano, su utilidad es evidente con miras a perfeccionar la personalización. Así por ejemplo, para alguien que disfruta de las bebidas gaseosas y los álbumes de rock, será mucho más interesante la publicidad sobre una refrescante bebida durante el tiempo que hace ejercicio.

Finalmente, es importante destacar algunos frameworks basados en técnicas de aprendizaje automático y minería de datos, que pueden ser útiles al momento de construir un SR para diferentes dominios como Apache Mahout [53], Lenskit Ekstrand et al [54] y Weka [55].

\subsection{APLICACIONESN-SCREENYMIDDLEWARE DE COOPERACIÓN SMART TV - SMARTPHONE}

Como se dijo anteriormente, el naciente modelo de Smart TV expone un potencial interesante para extender las capacidades de interacción de las pantallas publicitarias convencionales tanto en el campo de 
la señalización digital como en el de los avisos publicitarios del televidente clásico, gracias a su capacidad de conexión con Smartphones o tabletas. Plataformas como MOVL [56] y Zeebox [57] han dado los primeros pasos para facilitar la interacción entre Smartphones y Smart TV; la primera define un modelo de conexión en la nube para este tipo de dispositivos y la segunda promete revolucionar el contexto de la televisión a través de la interacción con las redes sociales Twitter y Facebook usando clientes móviles, abriendo las puertas hacia un nuevo paradigma conocido como aplicaciones de múltiples pantallas ( $n$-screen). En términos simples, el paradigma de múltiples pantallas busca tomar ventaja de las capacidades de cada pantalla para desplegar el contenido de la mejor manera en cada una de ellas, más en un esquema de cooperación, que en una simple réplica de información. En el contexto de la publicidad ubicua, los beneficios pueden ser claramente perceptibles si se tiene en cuenta escenarios de pantallas públicas (public displays) en los cuales la información desplegada en el Smart TV está dirigida al contexto grupal, mientras que la pantalla de los Smartphones es usada con fines de personalización Yoon et al [58]. Así por ejemplo, los usuarios podrían estar recibiendo avisos publicitarios personalizados en su teléfono, relacionados con algún tipo de contenido que observan en la pantalla.

Al respecto, tradicionalmente varios trabajos de investigación se han enfocado en mecanismos de interacción entre las pantallas y los Smartphones a través de tecnologías de corto alcance como NFC o Bluetooth [59-61]. No obstante, en el marco de la publicidad ubicua es importante la existencia de un middleware que habilite no sólo la cooperación entre el Smart TV y los Smartphones, sino también el acceso a información contextual que pueda derivarse a partir de los mismos. En primera instancia, los trabajos de [8-7] realizan una caracterización más profunda de los entornos de señalización digital, proporcionan algunas guías de diseño de este tipo de espacios y plantean algunas consideraciones importantes que fijan un punto de partida relevante al momento de definir los requerimientos de un middleware de cooperación Smart TV - Smartphone con fines publicitarios. En el mismo sentido, Strohbach et al [62] realiza algunos planteamientos relevantes para la gestión del contexto en entornos de publicidad ubicua y realiza una revisión de sobre algunas plataformas que pueden habilitar el acceso a la información de los sensores y del contexto en general a través de los dispositivos móviles; [6]-[63] complementa el trabajo anterior planteando una arquitectura de referencia que involucra clientes móviles con propósitos publicitarios. De manera similar, otros trabajos [6467] han explorado la interacción de Smartphones con pantallas en el marco del paradigma de múltiples pantallas, pero aún la exploración es incipiente para el caso de Smart TV y particularmente para entornos de publicidad ubicua.

De otro lado, uno de los parámetros más importantes de la publicidad es la evaluación de los resultados teniendo en cuenta el 
precepto: "lo que es susceptible de medir, es susceptible de mejorar" Zaichkowsky [5]. Tradicionalmente, las medidas de audiencia en medios como la televisión responden a la medición de aspectos fundamentalmente demográficos que son reunidos a través de encuestas telefónicas o dispositivos especializados para entregar datos sobre los canales que están observando los televidentes. Igualmente, las pantallas públicas que se pueden encontrar en los grandes centros comerciales, pueden usar mecanismos alternos como cámaras especializadas en el registro de las visitas de los transeúntes [68-70]. Un modelo de cooperación Smart TV - Smartphone, no sólo ofrece la posibilidad de replicar algunas de las estrategias de medición de los modelos online (conteo de clics o seguimiento a los contenidos de interés del usuario), sino que también amplía sus potencialidades para los mismos usuarios a través de mecanismos de interacción que les permita realizar comentarios sobre los productos en un contexto social o hacer el seguimiento a sus propias preferencias gracias a la información disponible a través del TV o el Smartphone; esta interacción podría ser seguida por las compañías con fines de medición [13], [6].

\subsection{GESTIÓN DE PRIVACIDAD CON FINES PUBLICITARIOS}

Sin lugar a dudas, la privacidad de los usuarios al momento de acceder a la información de sus perfiles o incluso del contexto en el cual se encuentran con el ánimo de fortalecer el impacto de los anuncios publicitarios, no sólo es un aspecto crítico en los entornos de publicidad ubicua, sino que ha sido su verdadero talón de Aquiles. Algunos estudios, han encontrado que un buen porcentaje de usuarios rechaza la idea de ser rastreados con fines publicitarios Turow [71], aún cuando esto signifique obtener ofertas más personalizadas [7274]. Recientemente, redes sociales como Facebook son cuestionadas por el uso que pueda darse a la información que se encuentra en su poder $y$ reiteradamente se persuade a sus gestores para que revisen las políticas al respecto, lo cual refleja la preocupación y la importancia que dan los usuarios a la privacidad de su información Coursey [75]. Aproximaciones similares son usadas por gigantes de la industria como Apple, Google o Microsoft en cuanto a la gestión de anuncios y aunque existen algunas soluciones para el manejo de cookies y alternativas de rastreo en general, éstas usualmente requieren un análisis detallado de la relación costo/ beneficio Freudiger [76].

Como antecedentes, en el 2009 la Comisión Federal de Comercio - FTC (US. Federal Trade Comission) emitió algunas guías auto regulatorias para el comportamiento de la publicidad online FTC [72], como un esfuerzo para proteger la privacidad del consumidor en ausencia de una legislación más estricta Boucher [77]. En el mismo sentido, la Asociación Internacional para el Comercio al por Menor - POPAl (Point of Purchase Association International), liberó en el 2010 la primera versión de un conjunto de lineamientos de privacidad para el caso específico de señalización digital Smith [78]; 
aunque el código de POPAl es un punto de partida interesante para la auto-regulación de la industria al respecto, realmente no articula un conjunto completo de prácticas de información justa ni establece un marco de trabajo estricto de privacidad para las compañías dedicadas a la señalización digital. El lanzamiento de este tipo de iniciativas implica un mensaje claro para que las compañías y agencias de publicidad incorporen la gestión de privacidad en sus modelos actuales de negocio y de gestión de información; en la práctica, puede ser menos costoso integrar los controles de privacidad sobre tecnologías emergentes como en el caso de la publicidad ubicua que hacerlo sobre sistemas existentes y consolidados Geiger [79].

Porotrolado, algunostrabajos han planteado soluciones aplicables a contextos de publicidad ubicua orientados principalmente a teléfonos móviles. Adnostic Toubiana et al [80] y Privad Guha et al [81], han diseñado sistemas dirigidos a los navegadores para descargar los anuncios relevantes de forma offline y desplegarlos en momentos apropiados; Komulainen et al [82] ha propuesto un sistema de privacidad basado en permisos, en el cual la información del perfil es proporcionada por el consumidor y actualizada en la base de datos central; igualmente, la arquitectura del sistema MobiAd propuesta en Haddadi et al [83], considera un marco de referencia básico para la gestión de privacidad en entornos de telefonía móvil, en el cual persisten los desafíos de seguridad y privacidad inherentes a este tipo de tecnología. No obstante, aún se nota la ausencia de estudios que especifiquen claramente cuándo, cómo y dónde los consumidores están dispuestos a ser parte de la publicidad móvil. Al respecto, una reflexión interesante, es que se ha encontrado que aunque la privacidad es una preocupación generalizada para la mayoría de los usuarios, ésta podría ser potencialmente compensada monetariamente o a través del valor percibido por la oferta Haddadi et al [6]. En un momento dado, un almacén de ropa por ejemplo, puede ofrecer cupones de descuento en la próxima compra de los usuarios a cambio del acceso a mayor información sobre su perfil personal.

En otro contexto, Geiger [79] plantea algunas consideraciones para la gestión de privacidad en entornos de señalización digital basadas en prácticas de información justa (FIP), que buscan ofrecer una guía para las compañías en cuanto al tratamiento de la información de los usuarios. En este sentido, Carolis [40] propone una aproximación para dirigir recomendaciones a un grupo de usuarios usando una pantalla no interactiva, a partir de los modelos individuales de los miembros que componen el grupo; en el contexto de la señalización digital, el ofrecimiento de anuncios publicitarios a grupos de personas más que a individuos particulares, puede constituir una ventaja en términos de la protección a la privacidad. Desde este punto de vista, el modelo de Smart TV sugiere un esquema de interacción enriquecido que podría contribuir en gran medida en dicha protección, si se tiene en cuenta que la información personalizada nunca será pública gracias a la posibilidad de extender el contenido a las pantallas de 
los Smartphones de los usuarios. En ese orden de ideas, el desarrollo de sistemas que desplieguen contenido publicitario de propósito más general en pantallas públicas, al tiempo que entregan anuncios más personalizados en el dispositivo móvil, es una interesante línea de investigación.

\subsection{PUBLICIDAD SOCIAL}

Sin lugar a dudas, las redes sociales juegan un rol importante en la vida cotidiana de las personas en la actualidad. Desde el punto de vista de la publicidad, el interés de los comerciantes en las redes sociales radica principalmente en la posibilidad de facilitar a los consumidores una discusión más abierta sobre sus productos, una forma moderna de la modalidad "de boca en boca" (Word-of-mouth - WOM), la cual ha sido reconocida como una de las fuentes más influyentes en la transmisión de información desde los inicios de la sociedad humana Duan et al [84]. En este sentido, un principio básico que guía las investigaciones en esta línea es que se ha demostrado que los consumidores prefieren reunir información sobre sus compras potenciales a través de amigos o comunidades virtuales [85-86]; se estima que 6 de cada 10 adultos en los Estados Unidos han realizado algún tipo de investigación online sobre los productos y servicios que compran y cerca de un $25 \%$ han publicado comentarios o revisiones sobre sus compras Jasen [87]. En el contexto de las redes sociales, los usuarios tienen acceso a la publicidad y la información de los vendedores básicamente de dos maneras: a través de los anuncios en los sitios o las páginas de fidelidad a la marca (fan pages), lo cual facilita una comunicación más directa entre oferentes y compradores.

Desde el punto de vista de la publicidad ubicua, es posible afirmar que existen dos grandes corrientes de investigación que resulta interesante abordar: en primera instancia, el enriquecimiento de los anuncios publicitarios a partir de la interacción social y en segunda instancia, el apoyo de la interacción en las redes sociales a los sistemas de recomendaciones que usan filtros basados en comunidad. Con respecto a la primera aproximación, algunos trabajos plantean la inclusión de nuevos tipos de avisos publicitarios que incluyen comentarios generados por los usuarios Ranganathan et al [13]; así mismo, el potencial que ofrecen redes como Gowalla, Foursquare y Facebook Places para brindar información de localización ha llamado la atención de otros investigadores: por ejemplo, [88-89] plantean la entrega de cupones o estímulos a los miembros que se registran en la localización de un tienda específica y Grove [90] ofrece estímulos similares a los usuarios de Foursquare que realizan visitas con más frecuencia.

De otro lado, otro campo de investigación fértil en este contexto está relacionado con la posibilidad de llevar las redes sociales a los escenarios de señalización digital; la posibilidad de incluir comentarios (quotes) de Facebook sobre una marca en particular en una pantalla pública por ejemplo, constituye un escenario de motivación para este tipo de trabajos. Al respecto, Spiegler et al [91] plantea un caso de estudio para el despliegue de comentarios de redes 
sociales en pantallas públicas de pequeñas tiendas en Suiza; una interesante conclusión de este trabajo es que la capacidad de los clientes para procesar estímulos textuales en un ambiente de compra es limitada, luego el uso de la información de redes sociales debe examinarse con cuidado en este tipo de entornos. En este sentido, las investigaciones sugieren que debido a la naturaleza de las interacciones en las redes sociales, la puntualidad del contenido es un aspecto crucial; la información requiere ser digerida, interpretada y consumida en el menor tiempo posible, puesto que retardos excesivos pueden invalidar el contexto Ferdinando et al [92].

Por otro lado, el uso de redes sociales para mejorar la efectividad de las recomendaciones es otro campo de investigación atractivo pero aún incipiente. Como se dijo anteriormente, la evidencia sugiere que las personas confían más en las recomendaciones de sus amigos que en las recomendaciones similares de individuos anónimos, lo cual constituye el principio básico de esta aproximación Sinha et al [93]. En este tipo de sistemas, la recomendación está basada en los ratings proporcionados por los amigos del usuario, aunque recientemente se ha considerado también las relaciones e interacciones sociales de los usuarios en la adquisición de datos Ricci et al [94]. Dada la naciente exploración que se tiene en este campo, los resultados de las investigaciones son aún diversos. [2526] reportan que las recomendaciones basadas en redes sociales no son más precisas que las derivadas a partir de los filtros colaborativos clásicos, excepto en casos especiales donde los ratings de los usuarios para un ítem específico son altamente controversiales, o cuando se usan como alternativa para solucionar los inconvenientes asociados a los "usuarios" o "ítems" nuevos (cold start). Otros trabajos han demostrado que en algunos casos, la adición de datos sociales a los sistemas tradicionales mejora los resultados de las recomendaciones con respecto a los datos de similitud de perfiles [95-96].

Recientemente, un ingrediente adicional se ha sumado a las investigaciones en este contexto: las redes de confianza; en términos simples, este concepto se refiere a una red social que expresa qué tanto confían los miembros de una comunidad entre sí. Algunos ejemplos de esta aproximación pueden evidenciarse en la red social Goldbeck's Film Trust o EOpinions.com Golbeck [25], las cuales mantienen una red de confianza al indagar en los usuarios acerca de las personas en las cuales confían. Los sistemas de recomendaciones basados en esta técnica, usan el conocimiento que se origina a partir de las relaciones de confianza, para brindar recomendaciones más personalizadas a través de operadores de propagación y agregación, que permiten a los usuarios recibir recomendaciones de ítems que han logrado una alta calificación por parte de los usuarios en su red o aún por las personas que hacen parte de las redes de confianza de estos individuos. Algunos trabajos relacionados pueden ser consultados en [97-99]. No obstante, aunque existen algunos trabajos en el contexto de la publicidad ubicua [100- 
102], la investigación aún es incipiente, lo cual no descarta su enorme potencial, más si se tiene en cuenta el enriquecimiento de los mecanismos de interacción que se puede lograr a través de un modelo de cooperación Smart TV - Smartphone. Así las cosas, las recomendaciones publicitarias que se despliegan en una pantalla por ejemplo, podrían derivarse no sólo a partir de la similitud entre los usuarios que la observan, sino también del nivel de confianza que pueda inferirse de sus vínculos sociales, al tiempo que reciben recomendaciones de sus amigos cercanos directamente en su teléfono móvil.

\section{CONCLUSIONES}

La publicidad ubicua marca un hito importante en la evolución de los mecanismos de promoción que tradicionalmente se han conocido en el área del mercadeo. La posibilidad de enriquecer los avisos publicitarios a través de la personalización, el involucramiento de la información del contexto y los mecanismos de interacción implícita a través de las capacidades de los Smartphones y Smart TV modernos, son algunos de los beneficios directos de este nuevo tipo de publicidad.

Los sistemas de recomendaciones para publicidad ubicua, constituyen uno de los campos núcleo de la investigación que actualmente se adelanta sobre esta área. Específicamente, la publicidad ubicua impone nuevos retos de investigación relacionados con la extensión del espacio bidimensional Usuario $\mathrm{x}$ Item tradicional, hacia un espacio Usuario $x$ Ítem $x$ Contexto y la generación de recomendaciones hacia entornos grupales más que individuales, puntualmente en escenarios de señalización digital (Digital Signage).

El naciente modelo de Smart TV promete revolucionar el concepto de la televisión moderna y por consiguiente el de la publicidad, si se tiene en cuenta que éste ha sido su canal masivo por excelencia. No obstante, es de especial relevancia el potencial de Smart TV en escenarios de señalización digital, donde la investigación aún es incipiente. La mayoría de los trabajos relacionados se han enfocado en la capacidad de interacción con pantallas públicas a través de tecnologías como Bluetooth o NFC, lo cual sugiere un campo fértil de investigación en nuevos mecanismos y middleware de interacción que tome ventaja de las capacidades de Smart TV, no sólo para enriquecer la promoción como tal, sino también para incorporar nuevos modelos de medidas de audiencia.

La privacidad es sin lugar a dudas uno de los talones de Aquiles de la publicidad ubicua y por lo tanto un campo de investigación relevante, que de hecho abarca aspectos legales que rebasan los aspectos meramente técnicos y en el que comienzan a darse algunas iniciativas para fijar reglas claras al respecto. En términos generales, los antecedentes sugieren que los usuarios están dispuestos a permitir el acceso a la información de sus perfiles, siempre y cuando se perciba un beneficio claro que va más allá de la simple personalización de ofertas. De otro lado, en el contexto 
de la señalización digital, la cooperación Smart TV - Smartphone ofrece una posibilidad interesante de protección a la privacidad, gracias al establecimiento de perfiles grupales públicos y perfiles individuales privados que son explotados a través de capacidades de múltiples pantallas (n-screen).

El campo de la publicidad social, se abre paso en el contexto moderno gracias a la enorme influencia de las redes sociales y un principio básico que establece que las personas prefieren reunir información sobre sus compras potenciales a través de amigos. En este sentido, el enriquecimiento de anuncios publicitarios con información derivada de la interacción social, incluso en escenarios de señalización digital, y el apoyo que pueda agregar la dinámica de las redes sociales a la generación de recomendaciones en sistemas que usan filtros basados en comunidad, son dos de las corrientes más fuertes de investigación que se vislumbran en este campo.

\section{AGRADECIMIENTOS}

Agradecimientos especiales a la Vicerrectoría de Investigaciones de la Universidad del Cauca y al Grupo de Ingeniería Telemática por el soporte brindado en el desarrollo del trabajo.

\section{REFERENCIAS}

[1] AMA - American Marketing Association. (2013). Marketing Power - American Marketing Association. Recuperado en junio de 2013, de http://www. marketingpower.com/Pages/default.aspx
[2] Müller, J., Alt, F. \& Michelis, D. (2011). Pervasive Advertising. Londres, Inglaterra: Springer, pp. 1-29.

[3] Kotler, P. \& Keller, K. L. (2012). Marketing management. Upper Saddle River, N.J., EE.UU.: Prentice Hall.

[4] Meffert, H. (2012). Marketing: Grundlagen marktorientierter Unternehmensführung. Berlín, Alemania: Gabler Verlag.

[5] Zaichkowsky, J. L. (1985.) Measuring the Involvement Construct. J. Consum. Res., 12(3), pp. 341-352.

[6] Haddadi, H.; Hui, P.; Henderson, T. \& Brown, I. (2011). Targeted Advertising on the Handset: Privacy and Security Challenges. En Müller, J., Alt, F., \& Michelis, D., (Eds.). Pervasive Advertising (119-137). Londres, Inglaterra: Springer.

[7] Stalder, U. (2011). Digital Out-of-Home Media: Means and Effects of Digital Media in Public Space. En Müller, J., Alt, F., \& Michelis, D., (Eds.). Pervasive Advertising (31-56). Londres, Inglaterra: Springer.

[8] Rui, J. \& Cardoso, J.C.S. (2011). Opportunities and Challenges of Interactive Public Displays as an Advertising Medium. En Müller, J., Alt, F., \& Michelis, D., (Eds.). Pervasive Advertising (139-157). Londres, Inglaterra: Springer.

[9] Jewett, F. (2011). Why Smart TV is the next big thing. Recuperado en junio de 2013, de http://www.uievolution. com/mobileconnect/Mobile_Connect_ June_2011.pdf 
[10] Müller, J., Alt, F. \& Michelis, D. (2011). Pervasive Advertising. Londres, Inglaterra: Springer, pp. 1-29.

[11] Weiser, M. (1999). The computer for the 21st century. Sigmobile Mob Comput Commun Rev, 3(3), pp. 3-11.

[12] Boll, S., Schmidt, A., Kern, D., Streng, S., \& Holleis, P. (2008). Magic Beyond the Screen. leee Multimed., 15(4), pp. 8-13.

[13] Ranganathan, A. \& Campbell, R. H. (2002). Advertising in a pervasive computing environment. En Proceedings of the 2nd international workshop on Mobile commerce. New York, NY, EE.UU, pp. 10-14.

[14] Fogg, B. J. (diciembre, 2002). Persuasive technology: using computers to change what we think and do. Ubiquity, 2002(12), pp. 89-120.

[15] Otero, N. \& Rui, J. (2009). Worth and Human Values at the Centre of Designing Situated Digital Public Displays. Int J Adv Pervasive Ubiquitous Comput, 1(4), pp. 1-13.

[16] Ashton, W. B. (1997). Keeping abreast of science and technology: technical intelligence for business. Columbus, $\mathrm{OH}$, EE.UU.: Battelle Press.

[17] Matheo-Software. (2013). Matheo Analyzer. Recuperado en enero de 2013, de http://www.matheo-software.com/ es/productos/matheo-analyzer.html

[18] Harzing A-W. (2013). Publish or Perish. Recuperado en enero de 2013, de http:// www.harzing.com/pop.htm
[19] Mahmood, T. \& Ricci, F. (2009). Improving recommender systems with adaptive conversational strategies. En Proceedings of the 20th ACM conference on Hypertext and hypermedia. Nueva York, NY, EE.UU., pp. 73-82.

[20] Resnick, P. \& Varian, H. R. (1997). Recommender systems. Commun Acm, 40(3), pp. 56-58.

[21] Burke, R. (2007). Hybrid Web Recommender Systems. En Brusilovsky, P., Kobsa, A. \& Nejdl, W. (Eds.). The Adaptive Web (377-408). Berlín, Alemania: Springer

[22] Ricci, F. (2011). Recommender systems handbook. Nueva York, NY, EE.UU.: Springer.

[23] Rashid, A. M., Albert, l., Cosley, D., Lam, S. K., McNee, S. M., Konstan, J. A. \& Riedl, J. (2002). Getting to know you: learning new user preferences in recommender systems. En Proceedings of the 7th international conference on Intelligent user interfaces. Nueva York, NY, EE.UU., pp. 127-134.

[24] Yu, K., Schwaighofer, A., Tresp, V., Xu, X. \& Kriegel, H.P. (2004). Probabilistic memory-based collaborative filtering. leee Trans. Knowl. Data Eng., 16(1), pp. 56-69.

[25] Golbeck, J. (2006). Generating predictive movie recommendations from trust in social networks. En Proceedings of the 4th international conference on Trust Management. Berlín, Alemania, pp. 93-104. 
[26] Massa, P. \& Avesani, P. (2004). TrustAware Collaborative Filtering for Recommender Systems. En Meersman, R. \& Tari, Z. (Eds.). On the Move to Meaningful Internet Systems 2004: CoopIS, DOA, and ODBASE (492-508). Berlín, Alemania: Springer.

[27] Pazzani, M. J. (diciembre, 1999). A Framework for Collaborative, ContentBased and Demographic Filtering. Artif. Intell. Rev., 13(5-6), pp. 393-408.

[28] Huang, Z., Chen, H. \& Zeng, D. (enero, 2004). Applying associative retrieval techniques to alleviate the sparsity problem in collaborative filtering. Acm Trans Inf Syst, 22(1), pp. 116-142.

[29] Symeonidis, P. (2008). Contentbased Dimensionality Reduction for Recommender Systems. En Preisach, C., Burkhardt, P.D.H., Schmidt-Thieme, P.D.L. \& Decker, P.D.R. (Eds.). Data Analysis, Machine Learning and Applications (619-626). Berlín, Alemania: Springer.

[30] Billsus, D. \& Pazzani, M. J. (1998). Learning Collaborative Information Filters. En Proceedings of the Fifteenth International Conference on Machine Learning. San Francisco, CA, EE.UU., pp. 46-54.

[31] Balabanović, M. \& Shoham, Y. (1997). Fab: content-based, collaborative recommendation. Commun Acm, 40(3), pp. 66-72.

[32] Basu, C., Hirsh, H., \& Cohen W. (1998). "Recommendation as classification: using social and content-based information in recommendation. En Proceedings of the fifteenth national/tenth conference on Artificial intelligence/Innovative applications of artificial intelligence. Menlo Park, CA, EE.UU., pp. 714-720.

[33] Li, Q. \& Kim, B.M. (2003). An approach for combining content-based and collaborative filters. En Proceedings of the sixth international workshop on Information retrieval with Asian languages - Volume 11, Stroudsburg, PA, EE.UU., pp. 17-24.

[34] Schein, A. I., Popescul, A., Ungar, L. H. \& Pennock, D. M. (2002). Methods and metrics for cold-start recommendations. En Proceedings of the 25th annual international ACM SIGIR conference on Research and development in information retrieval. Nueva York, NY, EE.UU., pp. 253-260.

[35] Spaeth, A. \& Desmarais, M. C. (2013). Combining Collaborative Filtering and Text Similarity for Expert Profile Recommendations in Social Websites. En Carberry, S., Weibelzahl, S., Micarelli, A. \& Semeraro, G. (Eds.). User Modeling, Adaptation, and Personalization (178189). Berlín, Alemania: Springer.

[36] Rongfei, J., Maozhong, J., \& Chao, L. (2010). A new clustering method for collaborative filtering. En 2010 International Conference on Networking and Information Technology (ICNIT), pp. 488-492.

[37] Kuflik, T., Berkovsky, S., Carmagnola, F., Heckmann, D. \& Krüger, A. (2009). Advances in Ubiquitous User Modelling: Revised Selected Papers. Berlín, Alemania: Springer. 
[38] Heckmann, D. \& Krueger, A. (2003). A User Modeling Markup Language (UserML) for Ubiquitous Computing. En Brusilovsky, P., Corbett, A. \& de Rosis, F. (Eds.). User Modeling 2003 (393-397). Berlín, Alemania: Springer.

[39] Hussein, T., Linder, T., Gaulke, W., \& Ziegler, J. (2010). A Framework and an Architecture for Context-Aware Group Recommendations. En Kolfschoten, G., Herrmann, T. \& Lukosch, S. (Eds.). Collaboration and Technology (121128). Berlín, Alemania: Springer.

[40] Carolis, B. D. (2011). Adapting News and Advertisements to Groups. En Pervasive Advertising, J. Müller, F. Alt, and D. Michelis, Eds. Springer London, pp. 227246.

[41] Partridge K. and Begole B., (2011) "Activity-Based Advertising," En Müller, J., Alt, F., \& Michelis, D., (Eds.). Pervasive Advertising (83-101). Londres, Inglaterra: Springer.

[42] Masthoff, J. (febrero, 2004). Group Modeling: Selecting a Sequence of Television Items to Suit a Group of Viewers. User Model. User-Adapt. Interact., 14(1), pp. 37-85.

[43] Jameson, A. (2004). More than the sum of its members: challenges for group recommender systems. En Proceedings of the working conference on Advanced visual interfaces, Nueva York, NY, EE.UU., pp. 48-54.

[44] Santos, R., Marreiros, G., Ramos, C., Neves, J. \& Bulas-Cruz, J. (2006). Multiagent Approach for Ubiquitous Group
Decision Support Involving Emotions. En Ma, J., Jin, H., Yang, L.T. \& Tsai, J.J.P. (Eds.). Ubiquitous Intelligence and Computing (1174-1185). Berlín, Alemania: Springer..

[45] O'Connor, M., Cosley, D., Konstan, J.A. \& Riedl, J. (2002). PolyLens: A Recommender System for Groups of Users. En Prinz, W., Jarke, M., Rogers, Y., Schmidt, K. \& Wulf, V. (Eds.). ECSCW 2001 (199-218). Países Bajos: Springer.

[46] McCarthy, J.F. \& Anagnost, T.D. (1998). MusicFX: an arbiter of group preferences for computer supported collaborative workouts. Proceedings of the 1998 ACM conference on Computer supported cooperative work, Nueva York, NY, EE.UU., pp. 363-372.

[47] Kabassi, K. (febrero, 2010). Personalizing recommendations for tourists. Telematics Informatics, 27(1), pp. 51-66.

[48] Ardissono, L., Goy, A., Petrone, G., Segnan, M. \& Torasso, P. (2003). Intrigue: Personalized recommendation of tourist attractions for desktop and hand held devices. Appl. Artif. Intell., 17(8-9), pp. 687-714.

[49] Kim, J.K., Kim, H.K., Oh, H.Y. \& Ryu, Y.U. (2010). A group recommendation system for online communities. Int. J. Inf. Manag., 30(3), pp. 212-219.

[50] Christensen, I.A. \& Schiaffino S. (2011). Entertainment recommender systems for group of users. Expert Syst. Appl., 38(11), pp. 14127-14135. 
[51] Crossen, A., Budzik, J. \& K. Hammond, J. (2002). Flytrap: intelligent group music recommendation. Proceedings of the 7th international conference on Intelligent user interfaces, Nueva York, NY, EE.UU., pp. 184-185.

[52] Chao, D. L., Balthrop, J. \& Forrest, S. (2005). Adaptive radio: achieving consensus using negative preferences. Proceedings of the 2005 international ACM SIGGROUP conference on Supporting group work, Nueva York, NY, EE.UU., pp. 120-123.

[53] Mahout. (2013). Apache Mahout: Scalable machine learning and data mining. Recuperado el 5 de julio de 2013, de http://mahout.apache.org/.

[54] Ekstrand, M.D., Ludwig, M., Kolb, J. \& Riedl, J.T. (2011). Lenskit: a modular recommender framework. Proceedings of the fifth ACM conference on Recommender systems, Nueva York, NY, EE.UU., pp. 349-350.

[55] Weka. (2013). Weka 3 - Data Mining with Open Source Machine Learning Software in Java. Recuperado el 5 de julio de 2013, de http://www.cs.waikato.ac.nz/ml/weka/.

[56] MOVL. (2013). MOVL. Recuperado el 5 de julio de 2013, de http://movl.com/.

[57] Zeebox. (2013). Zeebox - Get the free app. Recuperado el 5 de julio de 2013, de http://zeebox.com/.

[58] Yoon, C., Um, T. \& Lee, H. (2012). Classification of $\mathrm{N}$-Screen Services and its standardization. 2012 14th International Conference on Advanced Communication Technology (ICACT), pp. 597-602.
[59] Holleis, P., Broll, G. \& Böhm, S. (2010). Advertising with NFC. Workshop on Pervasive Advertising and Shopping, in conjunction with the 8th International Conference on Pervasive Computing (Pervasive 2010), Helsinki, Finlandia.

[60] Rui, J., Otero, N., Izadi, S. \& Harper, R. (2008). Instant Places: Using Bluetooth for Situated Interaction in Public Displays. leee Pervasive Comput., 7(4), pp. 52-57.

[61] Mahato, H., Kern, D., Holleis, P. \& Schmidt, A. (2008). Implicit personalization of public environments using bluetooth. $\mathrm{CHI}$ '08 Extended Abstracts on Human Factors in Computing Systems, Nueva York, NY, EE.UU., pp. 3093-3098.

[62] Strohbach, M., Bauer, M., Martin, M. \& Hebgen, B. (2011). Managing Advertising Context. En Müller, J., Alt, F., \& Michelis, D., (Eds.). Pervasive Advertising (185205). Londres, Inglaterra: Springer.

[63] Nguyen, Q.N. \& Hoang P.M. (2010). Push delivery of product promotion advertisements to mobile users. Proceedings of the Pervasive Advertising and Shopping 2010 Workshop, Helsinki, Finlandia.

[64] Otero, N. \& Rui, J. (2009). Worth and Human Values at the Centre of Designing Situated Digital Public Displays. Int. J. Adv. Pervasive Ubiquitous Comput., 1(4), pp. 1-13, 34.

[65] Santos, P., Ribeiro, F.R. \& Metrolho, J. (2012). Using pervasive computing technologies to deliver personal and public ads in public spaces. 2012 7th Iberian Conference on Information Systems and Technologies (CISTI), pp. 1-6. 
[66] Michelis, D. \& Send, H. (2009). Engaging Passers-by with Interactive Screens-A Marketing Perspective. Gl Jahrestagung, pp. 3875-3881.

[67] Kaasinen, A. \& Yoon, Y.I. (2012). Mobile advertising model in N-Screen environment for CSCW. 2012 7th International Conference on Computing and Convergence Technology (ICCCT), pp. 140-143.

[68] Rui, J. \& Soares, A.M. (2010). Towards new advertising models for situated displays. Proceedings of the 3rd workshop on Pervasive Advertising, Helsinki, Finlandia.

[69] May, M., Körner, C., Hecker, D., Pasquier, M., Hofmann, U. \& Mende, F. (2009). Modelling Missing Values for Audience Measurement in Outdoor Advertising Using GPS Data. Gl Jahrestagung, pp. 3993-4006.

[70] Schrammel, J., Mattheiss, E., Döbelt, S., Paletta, L., Almer, A. \& Tscheligi, M. (2011). Attentional Behavior of Users on the Move Towards Pervasive Advertising Media. En Müller, J., Alt, F., \& Michelis, D., (Eds.). Pervasive Advertising (287-307). Londres, Inglaterra: Springer.

[71] Turow, J., King, J., Hoofnagle, C.J., Bleakley, A. \& Hennessy, M. (2013). Americans Reject Tailored Advertising and Three Activities that Enable It by:: SSRN. Recuperado el 7 de julio de 2013, de http://papers.ssrn.com/sol3/papers. cfm?abstract_id=1478214.

[72] FTC. Federal Trade Commission. (febrero, 2009). Staff Report: "Self-
Regulatory Principles For Online Behavioral Advertising: Tracking, Targeting, and Technology" Recuperado el 7 de julio de 2013, de http://www.ftc.gov/os/2009/02/ P085400behavadreport.pdf.

[73] Wirespring. (2013). Digital signage networks must guarantee viewer privacy. Recuperado el 7 de julio de 2013, de http://www.wirespring. com/dynamic_digital_signage_and_ interactive_kiosks_journal/articles/ Digital_signage_networks_must_ guarantee_viewer_privacy-569.html.

[74] TruMedia. (2013). Facial Recognition Boards Will Never Record, Share Data - MediaBuyerPlanner. Recuperado el 7 de julio de 2013, de http://www. mediabuyerplanner.com/entry/34111/ trumedia-facial-recognition-boardswill-never-record-share-data/.

[75] Coursey, D. (2013). After Criticism, Facebook Tweaks Friends List Privacy Options, PCWorld. Recuperado el 7 de julio de 2013, de http://www.pcworld. com/article/184418/After_Criticism_ Facebook_Changes_Friend_List_ Privacy_Options.html.

[76] Freudiger, J., Vratonjic, N. \& Hubaux J.P. (2009) Towards privacy-friendly online advertising. IEEE Web 2.0 Security and Privacy (W2SP).

[77] Boucher, R. (2013). Behavioral ads: The need for privacy protection. Recuperado el 7 de julio de 2013, de http://thehill. com/special-reports/technologyseptember-2009/60253-behavioralads-the-need-for-privacy-protection. 
[78] Smith, G. (2013). Taking Consumer Privacy Seriously, POPAl's Digital Signage Group Releases Code of Conduct. Recuperado el 7 de julio de 2013, de http://www.popai.com/2010/02/08/ taking-consumer-privacy-seriouslypopais-digital-signage-group-releasescode-of-conduct/?cat_id=.

[79] Geiger, H.L. (2011). A Standard for Digital Signage Privacy. En Müller, J., Alt, F., \& Michelis, D., (Eds.). Pervasive Advertising (103-117). Londres, Inglaterra: Springer.

[80] Toubiana, V., Narayanan, A., Boneh, D., Nissenbaum, H. \& Barocas, S. (febrero, 2010). Adnostic: Privacy Preserving Targeted Advertising. NDSS.

[81] Guha, S., Reznichenko, A., Tang, K., Haddadi, H. \& Francis, P. (2009). Serving Ads from localhost for Performance, Privacy, and Profit. HotNets.

[82] Komulainen, H., Ristola, A. \& Still, J. (2006). Mobile advertising in the eyes of retailers and consumers - empirical evidence from a real-life experiment. International Conference on Mobile Business. ICMB '06, pp. 37-37.

[83] Haddadi, H., Hui, P. \& Brown, I. (2010). MobiAd: private and scalable mobile advertising. Proceedings of the fifth ACM international workshop on Mobility in the evolving internet architecture, Nueva York, NY, EE.UU., pp. 33-38.

[84] Duan, W., Gu, B. \& Whinston, A. B. (2008). The dynamics of online word-ofmouth and product sales-An empirical investigation of the movie industry. J. Retail., 84(2), pp. 233-242.
[85] Wang, M.C.H., Wang E.S.T., Cheng, J.M.S. \& Chen, A.F.L. (2009). Information quality, online community and trust: a study of antecedents to shoppers' website loyalty. Int. J. Electron. Mark. Retail., 2(3), pp. 203-219.

[86] Jepsen, A.L. (2006). Information Search in Virtual Communities: Is it Replacing Use of Off Line Communication?. J. Mark. Commun., 12(4), pp. 247-261.

[87] Jasen, J. (2013). Online Product Research. Pew Research Center's Internet \& American Life Project. Recuperado el 7 de julio de 2013, de http://www. pewinternet.org/Reports/2010/OnlineProduct-Research.aspx.

[88] Ailawadi, K. L., Beauchamp, J. P., Donthu, N., Gauri, D. K., \& Shankar, V. (2009). Communication and Promotion Decisions in Retailing: A Review and Directions for Future Research. J. Retail., 85(1), pp. 4255.

[89] Bustos, L. (2013). 110 Ways Retailers are Using Social Media Marketing. Recuperado el 7 de julio de 2013, de http://www.getelastic.com/socialmedia-examples/.

[90] Grove, J.V. (2013). Mayors of Starbucks Now Get Discounts Nationwide with Foursquare. Recuperado el 7 de julio de 2013, de http://mashable. com/2010/05/17/starbucks foursquare-mayor-specials/.

[91] Spiegler, E.D., Hildebrand, C. \& Michahelles, F. (2011). Social Networks in Pervasive Advertising and Shopping. En Müller, J., Alt, F., \& Michelis, D., 
(Eds.). Pervasive Advertising (207-225). Londres, Inglaterra: Springer.

[92] Ferdinando, A.Di., Rosi, A., Lent, R., Manzalini, A. \& Zambonelli, F. (2009). MyAds: A system for adaptive pervasive advertisements. Pervasive Mob. Comput., 5(5), pp. 385-401.

[93] Sinha, R.R. \& Swearingen, K. (2001). Comparing Recommendations Made by Online Systems and Friends. DELOS workshop: personalisation and recommender systems in digital libraries, vol. 106.

[94] Ricci, F., Rokach, L. \& Shapira, B. (2011). Introduction to Recommender Systems Handbook. En Ricci, F., Rokach, L., Shapira, B. \& Kantor, P.B. (Eds.). Recommender Systems Handbook. Nueva York, NY, EE.UU: Springer. pp. 1-35.

[95] Groh, G. \& Ehmig, C. (2007). Recommendations in taste related domains: collaborative filtering vs. social filtering. Proceedings of the 2007 international ACM conference on Supporting group work, Nueva York, NY, EE.UU., pp. 127-136.

[96] Guy, I., Zwerdling, N., Carmel, D., Ronen, I., Uziel, E., Yogev, S. \& Ofek-Koifman, S. (2009). Personalized recommendation of social software items based on social relations. Proceedings of the third ACM conference on Recommender systems, Nueva York, NY, EE.UU., pp. 53-60.

[97] Avesani, P., Massa, P. \& Tiella, R. (2005). Moleskiingit:atrust-awarerecommender system for ski mountaineering. Int. J. Infonomics, vol. 20.
[98] O'Donovan, J. \& Smyth, B. (2005). Trust in recommender systems. Proceedings of the 10th international conference on Intelligent user interfaces, Nueva York, NY, EE.UU., pp. 167-174.

[99] Victor, P., Cornelis, C., De Cock, M. \& Teredesai, A. M. (2008). Key figure impact in trust-enhanced recommender systems. Ai Commun, 21(2-3), pp. 127143.

[100] Clemons, E. K., Barnett, S. \& Appadurai, A. (2007). The future of advertising and the value of social network websites: some preliminary examinations, Proceedings of the ninth international conference on Electronic commerce, Nueva York, NY, EE.UU., pp. 267-276.

[101] Yang, W.S. \& Dia, J.B. (2008). Discovering cohesive subgroups from social networks for targeted advertising. Expert Syst. Appl., 34(3), pp. 2029-2038.

[102] Yang, W.S., Dia, J.B., Cheng, H.C. \& Lin, H.T. (2006). Mining Social Networks for Targeted Advertising. Proceedings of the 39th Annual Hawaii International Conference on System Sciences, 2006. HICSS '06, 6, p. 137a. 